\title{
Geometric phase in NMR interferometry experiment
}

\author{
By N. R. SKRYNNIKOV and B. C. SANCTUARY \\ Department of Chemistry, McGill University, 801 Sherbrooke Street West, \\ Montreal, Canada H3A 2K6
}

(Received 21 June 1994; accepted 24 August 1994)

\begin{abstract}
Experimental detection of geometric phase effects is based on interferometry techniques. The observation of a nonadiabatic geometric phase in nuclear magnetic resonance has been reported by D. Suter, K. T. Mueller, and A. Pines (1988, Phys. Rev. Lett., 60, 1218). A rigorous interpretation of their results is presented here. The experiment exploits the concept of fictitious spin-1/2 subsystems embedded in a spin system of higher order. A relevant expression for the geometric phase is derived here using the Euler rotation representation for the SU(2) group. In order to correlate the experimental data to the geometric phase, a decomposition of the density matrix in a basis of pure states is used and the isomorphism between the cyclic evolution of pure states and mixed states is constructed. Rigorous treatment of the dynamic phase, along with technical aspects of the experiment, shows that the observed echo shift is related directly to the geometric phase, in agreement with Suter et al. The observed geometric phase is classified as a frame-related phase.
\end{abstract}

\section{Introduction}

The discovery of the topological phase by Berry [1], followed by fundamental generalization due to Aharonov and Anadan [2], initiated intense experimental studies aimed at the detection of the geometric phase. To mention only a few, this included neutron interferometry [3], nonlinear optics [4] and electron diffraction studies [5].

Considerable effort was applied in the field of magnetic resonance and related areas. The adiabatic (Berry's) phase has been the subject of study by Pines' group [6] and its manifestation in magic-angle spinning experiments has been examined by Gan and Grant [7]. The non-Abelian Berry's phase was investigated in a nuclear quadrupole resonance experiment by Tycko [8] with the refined interpretation proposed recently by Kwon, Kim and Kim [9]. The connection with Bloch-Siegert shift was drawn by Furman and Kadzhaya [10]. Finally, Berry's phase effects in the context of dissipative evolution in electron spin resonance were discussed by Gamliel and Freed [11]. It should be noted that all of the forementioned magnetic resonance studies are confined to the case of adiabatic evolution. In contrast, the experiment due to Suter, Mueller and Pines [12] (SMP experiment) deals with the nonadiabatic geometric phase, which should be regarded as a more general concept [2].

The original communication which reports the SMP experiment [12] contains the physical basis for an understanding of the observed geometric phase effect. Experiments were performed over a three level spin system which shows a simple doublet spectrum. The magnetization vector associated with one pair of levels was forced into a cycle of evolution along a closed circuit on the Bloch sphere. The accumulated phase shift, containing the geometric part, was detected with the help 
of a spin-echo sequence applied to the other pair of levels. Such indirect detection is possible due to the common level shared by two transitions.

The goal of this paper is to provide for a rigorous mathematical interpretation of the SMP experiment. The points of particular interest include the correlation between the geometric phase exhibited by pure states and mixed states, as well as the problem of invariance of the geometric phase under a rotating frame transformation. The approach presented here is convenient for treating nuclear magnetic resonance (NMR) interferometry experiments since it allows for accurate separation of the two evolving subsystems within a spin density matrix.

The basis for the concept of the geometric phase is constituted in the fact that the Hilbert space of a quantum mechanical system can be expressed locally as the direct product $M \otimes \mathrm{U}(1)$, where $\mathrm{U}(1)$ is associated with the phase coordinate (Abelian phase is implied here). The union of subsets $M$ forms a projective Hilbert space. Cyclic evolution curves embedded in $M$ give rise to a multiplicative phase factor which includes the part determined by the geometry of the curves. Judicious choice of the projective Hilbert space is, we believe, a key point in geometric phase calculations.

One choice available for a periodic Hamiltonian is based on the application of Floquet theory. The coordinate system, implanted into a projective Hilbert space, is represented by an $n \times n$ unitary matrix. Cyclic evolution paths are given by periodic matrices $S(t)$ which can be found from the Floquet decomposition of the evolution operator [13].

Another choice is available for Hamiltonians possessing Lie algebraic properties [14-16]. This approach, applied in our analysis, facilitates the construction of a compact projective Hilbert space coordinatized by raising (lowering) operators of the algebra. It is shown below how the solid-angle result for the geometric phase can be derived in a concise manner using an appropriate representation of the SU(2) group (cf. reference [17]). Explicit forms of evolution paths in projective Hilbert space can be found by solving systems of differential equations which follow from the Schrödinger equation.

\section{Spin evolution under SU(2) Hamiltonian}

Consider the evolution of a quantal system under the Hamiltonians

$$
H(t)=\omega_{x}(t) I_{x}+\omega_{y}(t) I_{y}+\omega_{z}(t) I_{z} .
$$

This possesses the simple Lie algebra structure of $\mathrm{su}(2)$ and, therefore, the evolution operator constitutes an element of the group $G=\mathrm{SU}(2)$, allowing for a variety of exponential representations [18]. We opt for the representation which is used for generating Wigner rotation matrices. This was applied to the analysis of the geometric phase in rotating systems by Cui [19]. The representation is given by

$$
D(\alpha, \beta, \gamma)=\mathrm{e}^{i \gamma(t) I_{z}} \mathrm{e}^{i \beta(t) I_{y}} \mathrm{e}^{i \alpha(t) I_{z}} .
$$

Evidently, the evolution operator corresponding to Hamiltonian (1) falls in the class defined by equation (2), $U\left(t, t_{0}\right)=D_{U}(\alpha, \beta, \gamma)$, with initial conditions given by $\beta\left(t_{0}\right)=0, \alpha\left(t_{0}\right)=-\gamma\left(t_{0}\right)$.

Assume a spin system is prepared initially in the form of a fully polarized beam containing spins of different sorts and modified subsequently in the course of the preparation period by the action of operator (2). The evolution of the state $\left|\psi_{m}\left(t_{0}\right)\right\rangle$ 
produced can be described using the group properties of rotations $D$ as

$$
\begin{aligned}
\left|\psi_{m}(t)\right\rangle & =U\left(t, t_{0}\right)\left|\psi_{m}\left(t_{0}\right)\right\rangle=D_{U}(\alpha, \beta, \gamma)\left[D\left(\alpha_{0}, \beta_{0}, \gamma_{0}\right) \sum_{j=j_{0}}^{j_{n}} w_{j}|j, m\rangle\right] \\
& =D\left(\alpha^{\prime}, \beta^{\prime}, \gamma^{\prime}\right) \sum_{j=j_{0}}^{j_{n}} w_{j}|j, m\rangle .
\end{aligned}
$$

The primes on the Euler angles are dropped in the following. The construction used for the initial state $\left|\psi_{m}\left(t_{0}\right)\right\rangle$ may seem to be artificial, but it is significant for establishing the connection between evolution of pure states and mixed states (see equation (16)). Ansatz (3) can be inserted in the Schrödinger equation with Hamiltonian (1), leading to a system of differential equations for $\alpha(t), \beta(t), \gamma(t)$ [20, $21]$.

Inspecting expression (3), it is noted that the phase increment acquired by the state during a cycle of evolution is associated entirely with the $\exp \left(i \alpha(t) I_{z}\right)$ term. In other words, the maximum isotropy subgroup [14] of $\mathrm{SU}(2)$ with respect to $\sum_{j=j_{0}}^{j_{n}} w_{j}|j, m\rangle$ coincides with its Cartan subgroup $\mathfrak{I}$, which is generated by a single operator $I_{z}$ and, in general, is parametrized by one angle $\alpha(t)$ only. However, in order to account for trajectories which commence at $\beta\left(t_{0}\right)=0$ (north pole), equation (3) must be put into a symmetric form:

$$
\left|\psi_{m}(t)\right\rangle=D(-\gamma, \beta, \gamma) \mathrm{e}^{(\alpha(t)+\gamma(t)) I_{z}} \sum_{j=j_{0}}^{j_{n}} w_{j}|j, m\rangle .
$$

This yields the representation of the coset space $G / \mathfrak{I}=\mathrm{SU}(2) / \mathrm{U}(1)$ in the form of $D(-\gamma, \beta, \gamma)$. The coset space $G / \mathfrak{I}$ can be identified as a realization of projective Hilbert space. It allows for the mapping onto a two-sphere, $\mathrm{SU}(2) / \mathrm{U}(1)=\mathrm{SO}(3) / \mathrm{SO}(2)=\mathrm{S}^{2}$. The geometric phase effects are then associated with closed trajectories in this projective Hilbert space which are specified, in general, by a pair of conditions $\beta\left(t_{0}+T\right)=\beta\left(t_{0}\right), \gamma\left(t_{0}+T\right)=\gamma\left(t_{0}\right)$. In the particular case that $\beta\left(t_{0}\right)=0$ cyclic trajectories need to be closed only in $\beta(t)$ as a consequence of the coordinate singularity. In this special case the minimum available realization of projective Hilbert space is a circle $\mathrm{S}^{1}$ parametrized by $\beta(t)$.

The results obtained are used to generate generalized coherent states $[14,15]$ or single valued cyclic states [2] which are used to calculate the geometric phase in the Aharonov-Anandan analysis:

$$
\left|\phi_{m}(t)\right\rangle=D(-\gamma(t), \beta(t), \gamma(t)) \sum_{j=j_{0}}^{j_{n}} w_{j}|j, m\rangle .
$$

The geometric phase can be found following the Aharonov-Anandan's prescription that involves the differentiation of $\left|\phi_{m}(t)\right\rangle$. Relevant relationships for the spin algebra obeying

can be formulated as

$$
\left[I_{k}, I_{l}\right]=i \epsilon_{k l m} I_{m}
$$

$$
\exp \left(-i \theta I_{\mu}\right) I_{\eta} \exp \left(i \theta I_{\mu}\right)=I_{\eta} \cos \theta+\epsilon_{\mu \eta v} I_{v} \sin \theta
$$

where $\epsilon_{k l m}$ is the Levi-Civita tensor. Processing the derivative by use of equation (7) and then taking the integral over the period of evolution yields the following 
result for the geometric phase:

$$
\begin{aligned}
\chi_{\text {Geom }}^{m}(T) & =i \int_{t_{0}}^{t_{0}+T}\left\langle\phi_{m}(t)\left|\frac{\mathrm{d}}{\mathrm{d} t}\right| \phi_{m}(t)\right\rangle \mathrm{d} t=m \int_{t_{0}}^{t_{0}+T}(1-\cos \beta(t)) \dot{\gamma}(t) \mathrm{d} t \\
& =\oint_{C}(1-\cos \beta(t)) \mathrm{d} \gamma .
\end{aligned}
$$

The final equality in equation (8) is written as the contour integral along a closed path $C$ on the surface of the two-sphere discussed above. It recovers, in a simple manner, the well known solid angle result for the geometric phase [17].

The evolution operator $U\left(t, t_{0}\right)$ is often treated with the help of unitary time dependent transformations, for example, invoking the interaction representations. In practice, there is no need to go beyond the class of transformations belonging to $\mathrm{SU}(2)$, denoted by $D_{\mathrm{T}}(\Omega)$ :

$$
U\left(t, t_{0}\right)=D_{\mathrm{T}}(\Omega(t)) \tilde{U}\left(t, t_{0}\right) D_{\mathrm{T}}^{-1}\left(\Omega\left(t_{0}\right)\right)=D_{\mathrm{T}}(\Omega(t)) D_{\tilde{\mathrm{U}}}(\tilde{\alpha}, \tilde{\beta}, \tilde{\gamma}) D_{\mathrm{T}}^{-1}\left(\Omega\left(t_{0}\right)\right) .
$$

The Euler angles $\tilde{\alpha}(t), \tilde{\beta}(t), \tilde{\gamma}(t)$ can be determined from the system of differential equations [20] following the Schrödinger equation with correspondingly transformed Hamiltonian:

$$
\tilde{H}(t)=D_{\mathrm{T}}^{-1}(\Omega(t)) H(t) D_{\mathrm{T}}(\Omega(t))-i D_{\mathrm{T}}^{-1}(\Omega(t)) \frac{\mathrm{d}}{\mathrm{d} t} D_{\mathrm{T}}(\Omega(t)) .
$$

Obviously, representation (9) preserves the results obtained for the evolving state, including the overall phase and its geometric component. Alternatively, the transformed evolution operator $\tilde{U}\left(t, t_{0}\right)$ can be chosen to describe the net evolution effects in the sense of equation (3). In doing so the results for the overall and geometric phases are reformulated in terms of $\tilde{\alpha}(t), \tilde{\beta}(t), \tilde{\gamma}(t)$.

Thinking of $D_{\mathrm{T}}(\Omega)$ as a coordinate frame transformation, we can refer to $\tilde{U}\left(t, t_{0}\right)$ as a frame-related evolution operator. The dynamics of the system under $\tilde{U}\left(t, t_{0}\right)$ are determined by the modified Hamiltonian $\tilde{H}(t)$ and, therefore, are different from the original dynamics. This is exemplified in classical mechanics by the appearance of fictitious forces in rotating systems. Hence, the description involving $D_{\mathrm{U}}(\tilde{\alpha}, \tilde{\beta}, \tilde{\gamma})$ leads to frame-dependent results for the overall phase and its geometric component [22-24].

Considering frame-related evolution, phase effects can be removed by choosing $D_{\mathrm{T}}(\Omega)=U\left(t, t_{0}\right)$, which corresponds to a choice of the frame where the evolving state remains constant. In this case the trajectory $\{\tilde{\beta}(t), \tilde{\gamma}(t)\}$ collapses into a point, killing the geometric phase as well as the overall phase. On the other hand, it is always possible to provide for a transformation which preserves the overall phase, but makes the geometric phase nil. It can be stated, in this situation, that the geometric component is shifted into the dynamic part of the overall phase. For the special case of $\beta\left(t_{0}\right)=0$ this can be achieved by means of $D_{\mathbf{T}}(\Omega)=D(-\gamma, \beta, \gamma)$.

Consider the example of the rotating frame transformation which is involved in the concept of an SMP experiment. The transformation is given by

$$
D_{\mathrm{T}}(\Omega(t))=D(\omega t, 0,0)=\exp \left(i \omega t I_{z}\right) .
$$

Inserting this into equation (9) and putting $t_{0}=0$ yields immediately $\tilde{\alpha}=\alpha, \tilde{\beta}=\beta$, $\tilde{\gamma}=\gamma-\omega t$. Hence, the geometric phase in the laboratory frame $\chi_{\text {Geom }}^{m}(T)$, equation 
(8), is related to the rotating frame geometric phase $\tilde{\chi}_{\text {Geom }}^{m}(T)$ by

$$
\chi_{\text {Geom }}^{m}(T)=\tilde{\chi}_{\text {Geom }}^{m}(T)+m \omega \int_{t_{0}}^{t_{0}+T}(1-\cos \beta(t)) \mathrm{d} t .
$$

As may be seen, the difference between the laboratory frame and the rotating frame results is nontrivial and it is not compensated for by 'rotating frame detection' as adopted in experimental NMR.

In the general case, cyclic trajectories are closed in $\beta(t), \gamma(t)$ and, therefore, the overall phase is unaffected by a rotating frame transformation. However, in the special case of $\beta\left(t_{0}\right)=0$ (which is of particular interest for the NMR experiment in question) the overall phases are related by

$$
\chi_{\text {Overall }}^{m}(T)=\tilde{\chi}_{\text {Overall }}^{m}(T)+m \omega T,
$$

as follows from equation (4). Evidently, in this situation the difference is absorbed by 'rotating frame detection', which is conventional for experimental NMR.

To this end let us consider a mixed state (density matrix) $\sigma(t)$ of a quantum mechanical ensemble. Cyclic evolution of mixed states was first considered by Uhlmann [25] who formulated the concept of parallel transport in operator space using amplitude and phase factors attributed to the density matrix. This can be realized by decomposition of the density matrix in a basis of pure states, as has been pointed out by Dabrowski in his discussion of non-Abelian phases in model optical experiments [26]. This approach is exploited here allowing for reformulation of the density matrix evolution in terms of effective pure states. At this stage our goal is to construct the geometric image for mixed state evolution and to draw the connection between the trajectories of pure states and hypothetical trajectories of density matrices.

Consider the quantum system exposed to the Hamiltonian of equation (1). The evolution of the density matrix is given by the adjoint representation of $S U(2)$ :

$$
\sigma(t)=\operatorname{Ad}_{\mathbf{u}} \sigma\left(t_{0}\right)=U\left(t, t_{0}\right) \sigma\left(t_{0}\right) U^{-1}\left(t, t_{0}\right) .
$$

It is well known [27] that this adjoint representation specifies continuous homomorphism of $\mathrm{SU}(2)$ onto $\mathrm{SO}(3)$, Ad SU(2) $=\mathrm{SO}(3)$. The group $\mathrm{SO}(3)$ is generated by superoperators $\hat{I}_{x}, \hat{I}_{y}, \hat{I}_{z}$, which satisfy the commutation relationships (6). Thus, the group element can be represented as

$$
\hat{D}(\alpha, \beta, \gamma)=\exp \left(i \gamma(t) \hat{I}_{z}\right) \exp \left(i \beta(t) \hat{I}_{y}\right) \exp \left(i \alpha(t) \hat{I}_{z}\right) .
$$

Group $\mathrm{SO}(3)$ can be viewed as a restriction of SU(2) to the irreducible representation spaces of integer rank supplied with Cartan bases of spherical harmonics $Y_{m}^{(l)}$. Using the Cartan basis of spherical harmonics we introduce evolving mixed states in analogy with evolving pure states of equation (3):

$$
\sigma_{m}(t)=\hat{D}(\alpha, \beta, \gamma) \sum_{j=j_{0}}^{j_{n}} w_{j} Y_{m}^{(j)}
$$

It can be shown that the dynamics of mixed state (16) as expressed by $\alpha(t), \beta(t), \gamma(t)$ is equivalent to the dynamics of pure state (3) with Euler angles obeying one and the same system of differential equations. The differential equations can be derived by substitution of ansatz (16) into the quantum Liouville equation, followed by the application of superoperator commutation relationships analogous to (7) and 
spherical harmonics orthogonality. The extraction of the maximum isotropy subgroup associated with $\hat{I}_{z}$ can be carried out along the same lines, providing for a mapping onto $\mathrm{SO}(3) / \mathrm{SO}(2)=\mathrm{S}^{2}$.

The results obtained deserve some additional comments. Due to the Hermiticity of the density matrix, construct (16) allows for the value of $m=0$ only. This means that the initial states capable of cyclic evolution are produced by $\mathrm{SU}(2)$ transformation of the diagonal density matrix (cf. equation (3)). The equilibrium density matrix gives an example of the cyclic initial state. Furthermore, the overall phase, calculated for the density matrix evolving along the closed circuit, is nil. The density matrix treatment obviously can be extended to the case of frame-related evolution, and comments above concerning the special case of $\beta\left(t_{0}\right)=0$ also remain valid. It should be pointed out that the Euler angles involved in equation (16) are under experimental control and subject to direct measurements in an NMR experiment.

Finally, we derive the useful result which has been exploited in the paper of Suter, Mueller and Pines [12]. The operators $I_{x}, I_{y}, I_{z}$ can be combined into first-rank spherical tensor components $I_{m}^{(1)}$ giving rise to another realization of representation space of $\mathrm{SO}(3)$. By virtue of the irreducibility the Hamiltonian of equation (1) can be expressed using the adjoint representation of $I_{z}$ :

$$
H(t)=\omega \exp \left(i \xi \hat{I}_{z}\right) \exp \left(i \vartheta \hat{I}_{y}\right) \exp \left(i \hat{I}_{z}\right) I_{z} .
$$

Making use of equations (3) and (17), the criterion for the dynamic phase [2] to vanish is established as

$$
\cos \vartheta \cos \beta+\sin \vartheta \sin \beta \cos (\xi-\gamma)=0 .
$$

This condition corresponds to the orthogonality of two vectors: the first vector, as specified by $\{\beta(t), \gamma(t)\}$, traces the trajectory of the state on the surface of the sphere $\mathrm{S}^{2}$, whereas the second one $\{\vartheta(t), \xi(t)\}$ corresponds to a trajectory of Hamiltonian (17). Obviously, this observation remains valid in the case of frame-related evolution under $\tilde{H}(t)$.

\section{Interpretation of the Suter-Mueller-Pines experiment}

A pair of dipolar-coupled spin $1 / 2$ nuclei oriented in a liquid crystalline environment were chosen in the SMP experiment as a model system for demonstration of geometric phase effects in NMR. This system can be represented equivalently [28] by a spin $I=1$ subject to an axially symmetric quadrupolar interaction with the symmetry axis along the $z$ direction. It is often convenient to treat this system with the help of fictitious spin $1 / 2$ operators $[29,30]$.

The SMP experiment (figure 1) consists of a spin-echo sequence selectively applied to the high field transition with the consequent observation of an echo signal. Meanwhile, the magnetization associated with the low-field transition is forced into a cycle of evolution along the closed circuit. This was found to result in a phase shift of the echo signal correlated to the geometric phase.

Cyclic evolution of the low-field component has been realized by means of offset continuous wave $(\mathrm{CW})$ irradiation or pulse trains. These are generally referred to as a $\mathrm{C}$ sequence in the following. Since the duration of a $\mathrm{C}$ sequence is several times the duration of a $90^{\circ}$ pulse, we can disregard any relaxation effects in the course of a $\mathrm{C}$ sequence. Also the effects of irreversible relaxation are assumed negligible over the course of the whole experiment. Geometric phases in dissipative 
(C)
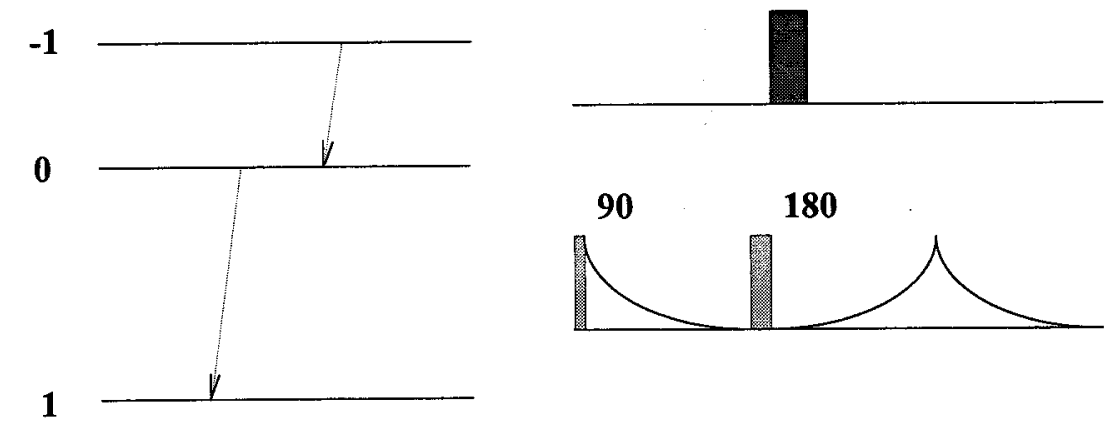

Figure 1. Experimental scheme of SMP [12]. The magnetization associated with the low-field transition is involved in a cycle of evolution through a specially engineered $\mathrm{C}$ sequence. The resulting phase shift is transferred onto the high-field transition via the common level, and is subsequently detected by means of the spin echo.

systems constitute a rather special case [31] which does not enter into the present anaysis.

In the following consideration we use the decomposition of a mixed state (density matrix) into a set of pure states [26]. This decomposition is non-unique and leads to stochastic evolution of pure states in the case when the interaction with the lattice is taken into account [32]. However, if this interaction is disregarded then there exists a well defined homomorphism between the mixed state representation and the pure state representation with respect to the quantum Liouville and Schrödinger equations.

The starting point of our consideration is the decomposition of the equilibrium density matrix into pure states. We describe an ensemble as a set of orthogonal pure states $\{|-1\rangle,|0\rangle,|1\rangle\}$ weighted with the probabilities $W_{-1}, W_{0}, W_{1}$ adjusted in such a way as to satisfy a Boltzmann distribution. At this point our goal is to keep track of the spin evolution up to the moment when the system is exposed to a $\mathrm{C}$ sequence. The effect of the first $90^{\circ}$ pulse is described readily by the use of a perturbation approach leading to the following mixture of pure states:

where

$$
\begin{aligned}
\left|\zeta_{-1}\right\rangle & =\exp \left(i \lambda_{-1} t\right)|-1\rangle \\
\left|\zeta_{0}\right\rangle & =(\sqrt{ } 2)^{-1} \exp \left(i \lambda_{0} t\right)|0\rangle-i(\sqrt{ } 2)^{-1} \exp \left(i \lambda_{1} t\right)|1\rangle \\
\left|\zeta_{1}\right\rangle & =-i(\sqrt{ } 2)^{-1} \exp \left(i \lambda_{0} t\right)|0\rangle+(\sqrt{ } 2)^{-1} \exp \left(i \lambda_{1} t\right)|1\rangle
\end{aligned}
$$

$$
\begin{aligned}
t & =\pi /\left(2(\sqrt{ } 2) \omega_{1}^{\mathrm{p}}\right) \\
\lambda_{m} & =m \omega_{0}+\left(m^{2}-2 / 3\right) \omega_{\mathrm{Q}}
\end{aligned}
$$

Here $\omega_{1}^{p}$ stands for the intensity of the RF pulse and $\omega_{Q}$ designates the effective quadrupolar splitting. Once again, the states (19.1-19.3) are weighted with $W_{-1}, W_{0}, W_{1}$.

In order to incorporate the effects of reversible relaxation, we assume that the magnetic field is inhomogeneous within a sample, which is expressed by the distribution $f(\delta \omega)$. Under these conditions the set of evolving vectors splits into a 'fan' of components $\left.\zeta_{m}(t, \delta \omega)\right\rangle$ weighted with the probabilities $W_{m} \cdot f(\delta \omega)$. The evolution is still given by equations (19), now with time $t$ playing a role of temporal variable and $\lambda_{m}$ replaced by $\lambda_{m}(\delta \omega)=\lambda_{m}+m \delta \omega$. 
In this situation it is easy to verify that the decay of the density matrix component $\rho_{0,1}(t)$, as generated by the set of vectors $(19.1-19.3)$, is given by the Fourier transform of $f(\delta \omega)$. The decay function corresponding to the observable free induction decay (FID) is real (i.e., does not incorporate a frequency shift) provided that $f(\delta \omega)$ is symmetrical with respect to $\omega_{0}$.

The effect of a $180^{\circ}$ pulse after a period of free evolution $\tau$ is determined similarly. Subsequent free precession is written as

$$
\begin{aligned}
\left|\zeta_{-1}(t, \delta \omega)\right\rangle= & \exp \left(i \lambda_{-1}(\delta \omega)[t-\tau]\right)|-1\rangle \\
\left|\zeta_{0}(t, \delta \omega)\right\rangle= & -(\sqrt{ } 2)^{-1} \exp \left(i \lambda_{1}(\delta \omega) \tau+i \lambda_{0}[t-\tau]\right)|0\rangle \\
& -i(\sqrt{ } 2)^{-1} \exp \left(i \lambda_{0} \tau+i \lambda_{1}(\delta \omega)[t-\tau]\right)|1\rangle \\
\left|\zeta_{1}(t, \delta \omega)\right\rangle= & -i(\sqrt{ } 2)^{-1} \exp \left(i \lambda_{1}(\delta \omega) \tau+i \lambda_{0}[t-\tau]\right)|0\rangle \\
& -(\sqrt{ } 2)^{-1} \exp \left(i \lambda_{0} \tau+i \lambda_{1}(\delta \omega)[t-\tau]\right)|1\rangle
\end{aligned}
$$

This gives rise to an echo signal centred at $t=2 \tau$. As expected, it recovers the original FID shape. Vectors (20) are used as the initial states for subsequent calculation of cyclic evolution under the $C$ sequence.

The initial state can be discussed also in terms of its density matrix as generated by vectors (20). From SMP, the transverse magnetization is seen to vanish at the moment when the $\mathrm{C}$ sequence is applied. This feature is provided for by the proper choice of $f(\delta \omega)$ in our model. Therefore, the initial density matrix is diagonal $\rho_{-1,-1}=W_{-1}, \rho_{0,0}=\rho_{1,1}=(1 / 2)\left(W_{0}+W_{1}\right)$, consistent with the fact that irreversible relaxation is absent.

Now we are in a position to investigate the effect of $\mathrm{C}$ sequence. The Hamiltonian corresponding to the $\mathrm{C}$ sequence can be written generally as

$$
\begin{aligned}
H(t)= & H_{0}+V(t)=-\omega_{0} I_{z}-\omega_{\mathrm{Q}}\left(I_{z}^{2}-2 / 3 E\right) \\
& +\omega_{1}(t)\left(\cos (\omega t+\varphi(t)) I_{x}-\sin (\omega t+\varphi(t)) I_{y}\right),
\end{aligned}
$$

where $\omega=\omega_{0}-\omega_{\mathrm{Q}}-\Delta$, with $\Delta$ playing the role of small offset, and where $\omega_{1}(t)$ and $\varphi(t)$ are the intensity and phase of the RF irradiation which vary from one pulse to another within the $\mathrm{C}$ sequence. By the argument of time-dependent perturbation theory [33], only on-resonance (or close to resonance) terms of $V(t)$ are retained, which leads to the matrix representation of $H(t)$ as

$$
H(t)=\left(\begin{array}{ccc}
(1 / 2)\left(\omega_{0}-\omega_{\mathrm{Q}}\right) & (\sqrt{ } 2)^{-1} \omega_{1}(t) \exp (-i(\omega t+\varphi(t))) & 0 \\
(\sqrt{ } 2)^{-1} \omega_{1}(t) \exp (i(\omega t+\varphi(t))) & (-1 / 2)\left(\omega_{0}-\omega_{\mathrm{Q}}\right) & 0 \\
0 & 0 & (-3 / 2)\left(\omega_{0}-\omega_{\mathrm{Q}} / 3\right)
\end{array}\right)+\eta E
$$

where

$$
\eta=(1 / 2)\left(\omega_{0}+\omega_{\mathrm{Q}} / 3\right) .
$$

The term $\eta E$, proportional to the identity $E$, has been extracted in such a way so as to make the upper block of the matrix traceless.

In view of the block-diagonal structure of $H(t)$, Hilbert space can be represented as a direct product of two uncoupled subspaces. One is the two-dimensional subspace spanned by $|-1\rangle,|0\rangle$, and the other is the one-dimensional subspace associated with 
vector $|1\rangle$. Projecting the vectors of equation (20) into these two subspaces yields

$$
\begin{aligned}
\left.\left|\zeta_{-1}(t, \delta \omega)\right\rangle\right|_{\mathrm{I}} & =\exp \left(i \lambda_{-1}(\delta \omega)[t-\tau]\right)|-1\rangle \\
\left.\left|\zeta_{0}(t, \delta \omega)\right\rangle\right|_{\mathrm{I}} & =\left.i\left|\zeta_{1}(t, \delta \omega)\right\rangle\right|_{\mathrm{I}}=-(\sqrt{ } 2)^{-1} \exp \left(i \lambda_{1}(\delta \omega) \tau+i \lambda_{0}[t-\tau]\right)|0\rangle \\
\left.\left|\zeta_{0}(t, \delta \omega)\right\rangle\right|_{\mathrm{II}} & =-\left.i\left|\zeta_{1}(t, \delta \omega)\right\rangle\right|_{\mathrm{II}}=-i(\sqrt{ } 2)^{-1} \exp \left(i \lambda_{0} \tau+i \lambda_{1}(\delta \omega)[t-\tau]\right)|1\rangle
\end{aligned}
$$

In the same manner, the density matrix can also be projected into these two subspaces.

At this point we can make the identification of the first subspace with a fictitious spin-1/2 space. Initial states of equation (23) can be regarded as polarized states of a spin-1/2 system, $\left|\psi_{1 / 2}\left(t_{0}\right)\right\rangle=w_{1 / 2}|1 / 2,1 / 2\rangle,\left|\psi_{-1 / 2}\left(t_{0}\right)\right\rangle=w_{1 / 2}^{\prime}|1 / 2,-1 / 2\rangle$, falling in the class $\left|\psi_{m}\left(t_{0}\right)\right\rangle=\sum_{j=j_{0}}^{j_{n}} w_{j}|j, m\rangle$ of equation (3). The initial density matrix for the fictitious spin-1/2 system can be represented in turn by $\sigma_{0}\left(t_{0}\right)=w_{0} Y_{0}^{(0)}+w_{1} Y_{0}^{(1)}$, belonging thereby to the class of equation $(16), \sigma_{0}\left(t_{0}\right)=\sum_{j=j_{0}}^{j_{n}} w_{j} Y_{0}^{(j)}$. The corresponding block of Hamiltonian (22) can be regarded as a spin-1/2 Hamiltonian, including a Zeeman splitting of $\omega_{0}-\omega_{\mathrm{Q}}$ and an RF irradiation of intensity $(\sqrt{ } 2) \omega_{1}(t)$. Obviously, a spin- $1 / 2$ system is formed in this way, which is embraced by our general description of spin dynamics under SU(2). Thus, all the results formulated in section 2 can be applied.

In the SMP experiment, the single transition density matrix $\sigma_{0}\left(t_{0}\right)$ is forced into a cycle of evolution along a closed trajectory on the surface of $\mathrm{S}^{2}$. A number of different cyclic trajectories (see figure 2) were generated by use of specially designed $\mathbf{C}$ sequences. As follows from the analysis presented in section 2 , the trajectories subtended by effective states $\left|\psi_{1 / 2}(t)\right\rangle,\left|\psi_{-1 / 2}(t)\right\rangle$ reproduce closed paths associated with $\sigma_{0}(t)$. Thus, the geometric phases accumulated by $\left|\psi_{1 / 2}\left(t_{0}\right)\right\rangle,\left|\psi_{-1 / 2}\left(t_{0}\right)\right\rangle$ are given by one-half of the solid angle enclosed by $\sigma_{0}(t)$ trajectories on the surface of the Bloch sphere. Note that the original dynamics of the system has been replaced in the SMP consideration by rotating frame dynamics. Therefore, the geometric phase obtained should be recognized as a frame-related phase.

The quantity which is related to the observable spin-echo phase shift is, in fact, the overall phase acquired by $\left|\psi_{1 / 2}\left(t_{0}\right)\right\rangle$. According to equation (13), it is expressed as

$$
\chi_{\text {Overall }}^{1 / 2}(T)=\tilde{\chi}_{\text {Dynam }}^{1 / 2}(T)+\tilde{\chi}_{\text {Geom }}^{1 / 2}(T)+(1 / 2) \omega T
$$

where $T$ is the duration of the $\mathrm{C}$ sequence. In order to determine the echo response it is necessary to combine the vector $\left|\psi_{1 / 2}\left(t_{0}+T\right)\right\rangle$ with the component evolving in the one-dimensional subspace $\left|\psi_{0}\left(t_{0}+T\right)\right\rangle$. This operation eliminates the phase shift arising from the $\eta E$ term in equation (22). Finally, in order to compare with the experimental data, detection in the rotating frame must be taken into account. This yields the result for the observable phase shift $\delta_{\mathrm{C}}$ induced on the echo signal due to the effect of the $\mathrm{C}$ sequence,

$$
\delta_{\mathrm{C}}=\tilde{\chi}_{\text {Dynam }}^{1 / 2}(T)+\tilde{\chi}_{\text {Geom }}^{1 / 2}(T)-(1 / 2) \Delta T
$$

This result can be discussed once the $\mathrm{C}$ sequence is specified. Different $\mathrm{C}$ sequences, as implemented in the SMP experiment, are presented in figure 2.

I. The trajectory 'slice' (figure $2(a)$ ). The trajectory is generated using a pair of $180^{\circ}$ on-resonance pulses, shifted by $\theta$ with respect to each other. The offset, $\Delta$, is 
(a)

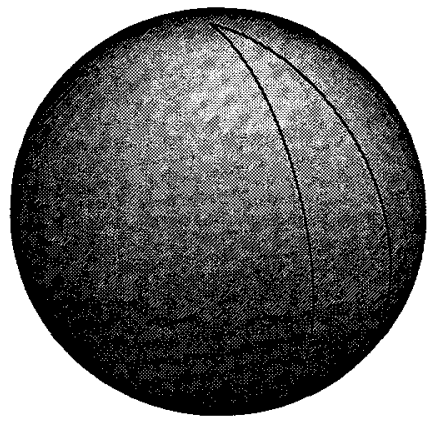

(b)

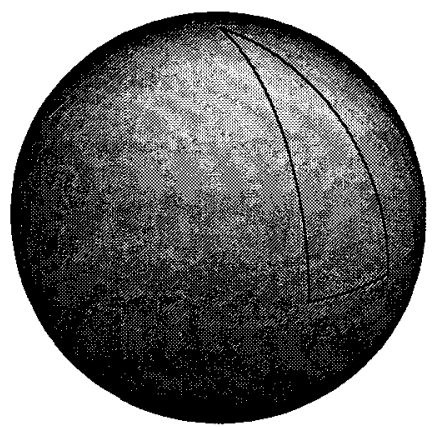

(c)

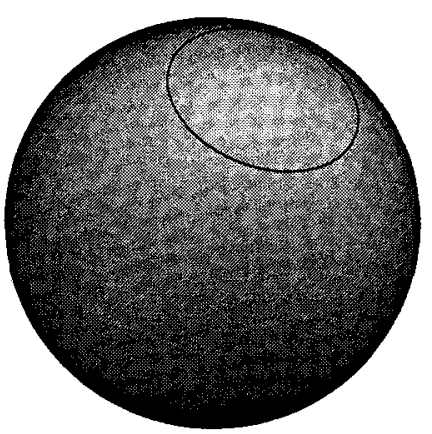

Figure 2. Different types of closed trajectory on the Bloch sphere representing the cyclic evolution of the single-transition density matrix, as realized in the SMP experiment: (a) slice, (b) triangle, and (c) cone.

kept zero for the duration of the experiment. As pointed out in the SMP communication, the dynamic phase vanishes along the trajectory due to the orthogonality of the vectors corresponding to the evolving state and the driving Hamiltonian (the condition is formulated in equation (18)). Thus, $\delta_{\mathrm{C}}=\tilde{\chi}_{\mathrm{Geom}}^{1 / 2}(T)$ or, recalling result (8), the observable shift is equal to one half the solid angle subtended by the trajectory. For the trajectory under consideration this implies $\delta_{\mathrm{C}}=\theta$, in agreement with experimental data.

II. The trajectory 'triangle' (figure $2(b)$ ). The trajectory is generated by use of a $90^{\circ}$ pulse, followed by a composite $z$ pulse, and then another $90^{\circ}$ pulse with appropriately adjusted phase. The composite $z$ pulse [34] consists of three onresonance selective pulses with effective net propagator of the form $\exp \left(i \theta I_{z}\right)$. In view of this propagator, the action of the $z$ pulse can be represented by an additional term $\omega_{z}(t) I_{z}$ introduced in the Hamiltonian of the fictitious spin-1/2 system (notice that a $z$ pulse acts selectively on the low-field transition). The time dependence of $\omega_{z}(t)$ reflects the fact that the $z$ pulse is active for a fraction of time $T, \int_{t_{0}}^{t_{0}+T} \omega_{z}(t) \mathrm{d} t=\theta$. The dynamic phase vanishes for $\left|\psi_{1 / 2}(t)\right\rangle$ by the argument of orthogonality, equation (18), including the part of the trajectory traversed under the effect of $\omega_{z}(t) I_{z}$. Consequently, the observable shift is found to be $\delta_{\mathrm{C}}=\tilde{\chi}_{\mathrm{Geom}}^{1 / 2}(T)=(1 / 2) \theta$, in agreement with the experiment results.

III. The trajectory 'cone' (figure $2(c)$ ). The trajectory is generated by offresonance irradiation of the low-field transition. The intensity of the RF field is constant, $\omega_{1}(t)=\omega_{1}$, and the RF phase $\varphi(t)$ is taken to be zero. The dynamic phase 
$\tilde{\chi}_{\text {Dynam }}^{1 / 2}(T)$ can be calculated using expression (3) for cyclic states along with the explicit solution for the Euler angles. This approach, however, proves to be tedious, since $\alpha(t), \beta(t), \gamma(t)$ are relatively complex functions in the present case [20]. Alternatively, we can build the cyclic state in a more tractable form by using a double-rotating frame representation which relies on a different type of exponential operator (it can be viewed also as the product of two Euler rotations):

$$
\left|\psi_{1 / 2}(t)\right\rangle=\exp \left(-i \tilde{\vartheta} I_{y}\right) \exp \left(-i \tilde{\Omega} t I_{z}\right) \exp \left(i \tilde{\vartheta} I_{y}\right)\left|\psi_{1 / 2}\left(t_{0}\right)\right\rangle
$$

where

$$
\begin{aligned}
& \tilde{\vartheta}=\operatorname{arctg}\left(\omega_{1} / \Delta\right) \\
& \tilde{\Omega}=\left(\Delta^{2}+\omega_{1}^{2}\right)^{1 / 2} .
\end{aligned}
$$

Applying the same differentiation rules as in section 2 to cyclic state (26), we can calculate the dynamic phase as defined by Aharonov and Anandan [2], $\tilde{\chi}_{\text {Dynam }}^{1 / 2}(T)=$ $(1 / 2) \Delta T$. Therefore, the observable shift is once again $\delta_{\mathrm{C}}=\tilde{\chi}_{\mathrm{Geom}}^{1 / 2}(T)$, being equal to one half of solid angle, which is expressed as [35]

$$
\delta_{\mathrm{C}}=\pi\left(1-\frac{\Delta}{\tilde{\Omega}}\right) .
$$

It is unnecessary to make any reference measurements in order to extract the geometric phase in this experiment. With this reservation, we can consider the theoretical prediction to be fully consistent with experiment results.

In conclusion, a three level spin system constitutes a framework for a NMR interferometry experiment. The isomorphism between $\mathrm{SU}(2) / \mathrm{U}(1)$ and $\mathrm{SO}(3) / \mathrm{SO}(2)$ allows direct experimental implementation of various closed trajectories for effective pure states on the Bloch sphere. The geometric phases acquired by effective states can be measured with the proviso that the dynamic phase disappears along the evolution path or, otherwise, is eliminated by rotating frame detection. Direct calculation of phases can be tedious due to the fact that the $\mathrm{C}$ sequence propagator does not commute with itself at different moments of time. However, the solid angle result for the geometric phase, deduced by use of the Euler angle parametrization, makes it possible to circumvent this difficulty by referring directly to the geometry of the evolution paths. The geometric phase observed in the Suter-Mueller-Pines experiment can be classified as frame-related, in contrast to the invariant Kobe's phase.

This work is supported by a grant from a Natural Sciences and Engineering Research Council of Canada (NSERC). We thank Dr D. Suter (ETH, Zürich) for clarification of experimental details and Dr J. Zhou (Wuhan Institute of Physics) for helpful discussions.

\section{References}

[1] Berry, M. V., 1984, Proc. R. Soc. Lond. A, 392, 45.

[2] Aharonov, Y., and Anandan, J., 1987, Phys. Rev. Lett., 58, 1593.

[3] WaGH, A. G., and Rakhecha, V. C., 1990, Phys. Lett. A, 148, 17.

[4] Chiao, R. Y., and Wu, Y. S., 1986, Phys. Rev. Lett., 57, 933.

[5] Bird, D. M., and Preston, A. R., 1988, Phys. Rev. Lett., 61, 2863.

[6] Suter, D., Chingas, G. C., Harris, R., and Pines, A., 1987, Molec. Phys., 61, 1327.

[7] Gan, Z.-H., and Grant, D. M., 1989, Molec. Phys., 67, 1419.

[8] Tyско, R., 1987, Phys. Rev. Lett., 58, 2281. 
[9] Kwon, O., Kim, S.-K., and Kim, Y., 1992, Phys. Lett. A, 164, 374.

[10] Furman, G. B., and Kadzhaya, I. M., 1993, J. magn. Reson. A, 105, 7.

[11] Gamliel, D., and Freed, J. H., 1989, Phys. Rev. A, 39, 3238.

[12] Suter, D., Mueller, K. T., and Pines, A., 1988, Phys. Rev. Lett., 60, 1218.

[13] Moore, D. J., 1990, J. Phys. A, 23, L665.

[14] Perelomov, A. M., 1986, Generalized Coherent States and Their Applications (Berlin: Springer-Verlag).

[15] Brihaye, Y., Giler, S., Kosiński, P., and Maślanka, P., 1990, J. Phys. A, 23, 1985.

[16] Cui, S.-M., 1993, Phys. Rev. A, 47, 690.

[17] GaO, X.-C., XU, J.-B., and Qian, T.-Z., 1991, Phys. Lett. A, 152, 449.

[18] Želobenko, D. P., 1973, Compact Lie Groups and their Representations (Providence, RI: American Mathematical Society).

[19] CuI, S.-M., 1992, Phys. Rev. A, 45, 5255.

[20] Zhou, J., GaO, H., and SAnctuary, B. C., 1993, J. magn. Reson. A, 101, 119.

[21] Cheng, C. M., and Fung, P. C. W., 1988, J. Phys. A, 21, 4115.

[22] KoBe, D. H., 1990, J. Phys. A, 23, 4249.

[23] Kendrick, B., 1992, J. Phys. A, 25, 885.

[24] Liang, J.-Q., and Müller-Kirsten, H. J. W., 1992, Ann. Phys., 219, 42.

[25] Uhlmann, A., 1986, Rep. Math. Phys., 24, 229.

[26] Dabrowski, L., 1991, Nuovo Cimento B, 106, 963.

[27] Sugiura, M., 1990, Unitary Representations and Harmonic Analysis (Amsterdam: North-Holland; Tokyo: Kodansha).

[28] Keller, A., 1988, Adv. magn. Reson., 12, 183.

[29] Wokaun, A., and ERnst, R. R., 1977, J. chem. Phys., 67, 1752.

[30] Vega, S., and Pines, A., 1977, J. chem. Phys., 66, 5624.

[31] Chu, S. I., Wu, Z. C., and Layton, E., 1989, Chem. Phys. Lett., 157, 151.

[32] Amann, A., 1993, Symposium on the Foundations of Physics: Quantum Measurement, Irreversibility and the Physics of Information, edited by P. Busch, P. J. Lahti and P. Mittelstaedt (Singapore: World Scientific).

[33] Landau, L. D., and LifShitz, E. M., 1977, Quantum Mechanics: Non-Relativistic Theory (Oxford: Pergamon Press).

[34] Freeman, R., Frenkiel, T. A., and Levitt, M. H., 1981, J. magn. Reson., 44, 409.

[35] Skrynnikov, N. R., Zhou, J., and Sanctuary, B. C., 1994, J. Phys. A, 27, 6253. 